International Journal of Pure and Applied Mathematics

Volume 93 No. 5 2014, 753-764

ISSN: 1311-8080 (printed version); ISSN: 1314-3395 (on-line version)

url: http://www.ijpam.eu

doi: http://dx.doi.org/10.12732/ijpam.v93i5.13

ijpam.eu

\title{
POISSON APPROXIMATION FOR THE NUMBER OF ISOLATED CYCLES IN A RANDOM INTERSECTION GRAPH
}

\author{
Mana Donganont \\ Department of Mathematics \\ School of Science, University of Phayao \\ Phayao, 56000, THAILAND
}

Abstract: Let $W_{n, k}$ be the number of isolated cycles of order $k$ in a random intersection graph $\mathbb{G}(n, m, p)$. In this paper, we demonstrate that $W_{n, k}$ can be approximated by Poisson distribution and give the bound of this approximation by using the Stein-Chen method.

Key Words: random intersection graph, isolated cycles, Stein-Chen method

\section{Introduction}

Given a set $V$ with $n$ vertices and another universal set $U$ with $m$ elements, define a bipartite graph $B(n, m, p)$ with independent vertex sets $V$ and $U$ and edges between $v \in V$ and $u \in U$ existing independently with probability $p$. The random intersection graph $\mathbb{G}(n, m, p)$, derived from $B(n, m, p)$, is defined on the vertex set $V$ with vertices $v_{1}, v_{2} \in V$ adjacent if and only if there exist some $u \in U$ such that both $v_{1}$ and $v_{2}$ are adjacent to $u$ in $B(n, m, p)$. Also define $S_{i}$ be a random subset of $U$ such that each element of $S_{i}$ is adjacent to $i \in V$, in which case two vertices $i, j \in V$ are adjacent if and only if $S_{i} \cap S_{j} \neq \phi$, and edge set $\mathrm{E}(\mathbb{G})$ is define as

$$
\mathrm{E}(\mathbb{G})=\left\{\{i, j\}: i, j \in V, S_{i} \cap S_{j} \neq \phi\right\} .
$$

The properties of $\mathbb{G}(n, m, p)$ were studied in $[2,3]$ contrasted with the well 
known random graph model $\mathbb{G}(n, p)$, in which vertices are made adjacent to each other independently and with probability $p$, and showed that for a fixed $\alpha>0$, the number of elements $m$ is taken to be $m=\left\lfloor n^{\alpha}\right\rfloor$. In 1999, Karonski, Scheinerman and Singer-Cohen[2] showed that the total variation distance between the distribution of $\mathbb{G}(n, m, p)$ and $\mathbb{G}(n, p)$ converges to 0 when $\alpha>6$ and $p$ is defined appropriately. Without loss of generality we consider the independent set $V$. For $i=1,2,3, \ldots, n$, let

$$
X_{i}= \begin{cases}1 & \text { if vertex } i \text { is an isolated in } \mathbb{G}(n, m, p) \\ 0 & \text { otherwise }\end{cases}
$$

and

$$
X=\sum_{i \in \Gamma} X_{i}
$$

Clearly, $X$ is the number of isolated vertices in $\mathbb{G}(n, m, p)$.

In 2011, Yilum Shang[6] proved that the distribution function of $X$ can be approximated by Poisson distribution with parameter

$$
\begin{aligned}
\lambda:=\mathrm{E} W=n P\left(X_{i}=1\right)=n \sum_{s=0}^{m}\left(\begin{array}{c}
m \\
s
\end{array}\right) p^{s}(1-p)^{m-s}(1-p)^{(n-1) s} & \\
& =n\left[1-p+p(1-p)^{(n-1)}\right]^{m} .
\end{aligned}
$$

In 2013, M. Donganont[9] showed the another proof of Poisson approximation for the number of isolated vertices in $\mathbb{G}(n, m, p)$ by Stein-Chen and coupling method. The results as the following,

Theorem $1.1(9)$. Let $W$ be the number of isolated vertices in a random intersection graph $\mathbb{G}(n, m, p)$.

For $A \subseteq\{0,1,2, \ldots n\}$ and $m=\left\lfloor n^{\alpha}\right\rfloor$ for some $\alpha>0$, we have

$$
\left|P(W \in A)-P \operatorname{Poi}_{\lambda}(A)\right| \leq \frac{n C_{\lambda, A}}{e^{p m\left(1-(1-p)^{n-2}\right)}}
$$

where $C_{\lambda, A}=\min \left\{1, \lambda, \frac{\triangle(\lambda)}{M_{A}+1}\right\}$,

$$
\triangle(\lambda)= \begin{cases}e^{\lambda}+\lambda-1 & \text { if } \lambda^{-1}\left(e^{\lambda}-1\right) \leq M_{A}, \\ 2\left(e^{\lambda}-1\right) & \text { if } \lambda^{-1}\left(e^{\lambda}-1\right)>M_{A},\end{cases}
$$

and

$$
M_{A}= \begin{cases}\max \left\{w \mid C_{w} \subseteq A\right\} & \text { if } 0 \in A, \\ \min \{w \mid w \in A\} & \text { if } 0 \notin A\end{cases}
$$

When $C_{w}=\{0,1, \ldots, w\}$ 
Corollary 1.1. [9] Let $W$ be the number of isolated vertices in a random intersection graph $\mathbb{G}(n, m, p)$. Let $A \subseteq\{0,1,2, \ldots n\}, m=\left\lfloor n^{\alpha}\right\rfloor$ for some $\alpha>0$, $q=1-p$, and $p=\frac{1}{n^{\gamma}}$ for any $\gamma \in \mathbb{R}^{+} \backslash\{1\}$, then

$$
\left|P(W \in A)-P o i_{\lambda}(A)\right| \leq \frac{C(\lambda, A)}{n^{2(\alpha-\gamma)\left(1-(q)^{n-2}\right)-1}},
$$

where $C(\lambda, A)=\min \left\{1, \lambda, \frac{\Delta(\lambda)}{M_{A}+1}\right\}$.

Let

$$
\Gamma_{n, k}=\left\{i=:\left\{i_{1}, i_{2}, \ldots, i_{k}\right\} \mid 1 \leq i_{1}<\ldots<i_{k} \leq n\right\}
$$

be the set of all possible combinations of $k$ vertices. we note that $T_{k}$ is a tree of order $k$ in $\mathbb{G}(n, m, p)$ and say that $T_{k}$ is isolated in $\mathbb{G}(n, m, p)$ if there is no edge in $\mathbb{G}(n, m, p)$ between a vertex in the tree and the other outside of the tree.

For each $i \in \Gamma_{n, k}$, we define the indicator random variable

$$
X_{i}=\left\{\begin{aligned}
1 & \text { if there is an isolated tree in } \mathbb{G}(n, m, p) \text { that spans the vertices } \\
& i=\left(i_{1}, \ldots, i_{k}\right), \\
0 & \text { otherwise }
\end{aligned}\right.
$$

and set

$$
W_{n, k}=\sum_{i \in \Gamma_{n, k}} X_{i} .
$$

Then $W_{n, k}$ is the number of isolated trees in $\mathbb{G}(n, m, p)$.

In 2013, Mana [10] shows that if $m=\left\lfloor n^{\alpha}\right\rfloor ; \alpha>0$, then $W_{n, k}$ can be approximated by Poisson approximation with parameter

$$
\begin{aligned}
\lambda:=\mathbb{E}\left(W_{n, k}\right)=\left(\begin{array}{l}
n \\
k
\end{array}\right) P\left(X_{i}=1\right) & =\left(\begin{array}{l}
n \\
k
\end{array}\right) \sum_{s=0}^{m}\left(\begin{array}{c}
m \\
s
\end{array}\right) p^{s+k-1}(1-p)^{m-s}(1-p)^{(n-k) s} \\
& =\left(\begin{array}{l}
n \\
k
\end{array}\right) p^{k-1}\left[1-p+p(1-p)^{(n-k)}\right]^{m}
\end{aligned}
$$

By using Stein-Chen and Coupling Method. The result is following,

Theorem $1.2(10)$. Let $W$ be the number of isolated trees in a random intersection graph $\mathbb{G}(n, m, p)$.

For $A \subseteq\{0,1,2, \ldots n\}$ and $m=\left\lfloor n^{\alpha}\right\rfloor$ for some $\alpha>0$, we have

$$
\left|P(W \in A)-P o i_{\lambda}(A)\right| \leq \frac{C_{(\lambda, A)}\left(1+\frac{n^{k}}{k !} p k^{2}\right) p^{k-1}}{e^{m p\left(1-(1-p)^{n-2 k}\right)}}
$$


where $C_{(\lambda, A)}=\min \left\{1, \lambda, \frac{\triangle(\lambda)}{M_{A}+1}\right\}$,

$$
\triangle(\lambda)= \begin{cases}e^{\lambda}+\lambda-1 & \text { if } \lambda^{-1}\left(e^{\lambda}-1\right) \leq M_{A} \\ 2\left(e^{\lambda}-1\right) & \text { if } \lambda^{-1}\left(e^{\lambda}-1\right)>M_{A}\end{cases}
$$

and

$$
M_{A}= \begin{cases}\max \left\{w \mid C_{w} \subseteq A\right\} & \text { if } 0 \in A \\ \min \{w \mid w \in A\} & \text { if } 0 \notin A\end{cases}
$$

When $C_{w}=\{0,1, \ldots, w\}$

Corollary 1.2. [10] Let $W$ be the number of isolated trees in a random intersection graph $\mathbb{G}(n, m, p)$. Let $A \subseteq\{0,1,2, \ldots n\}, m=\left\lfloor n^{\alpha}\right\rfloor$ for some $\alpha>0$, and $p=\frac{1}{n^{\gamma}}$ for any $\gamma \in \mathbb{R}^{+}\{1\}$

1. If $\gamma>k>0, q=1-p$, then

$$
\left|P(W \in A)-P \operatorname{Poi}_{\lambda}(A)\right| \leq \frac{C(\lambda, A)}{n^{2(\alpha-\gamma)\left(1-(q)^{n-2 k}\right)+(k \gamma-\gamma)}},
$$

where $C(\lambda, A)=2 \min \left\{1, \lambda, \frac{\triangle(\lambda)}{M_{A}+1}\right\}$.

2. If $k>\gamma>0, q=1-p$, then

$$
\left|P(W \in A)-\operatorname{Poi}_{\lambda}(A)\right| \leq \frac{C(\lambda, A)}{n^{2(\alpha-\gamma)\left(1-(q)^{n-2 k}\right)+(k \gamma-k)}},
$$

where $C(\lambda, A)=2 \min \left\{1, \lambda, \frac{\triangle(\lambda)}{M_{A}+1}\right\}$.

This work, we use this idea in order to show that the number of isolated cycles can be approximated by Poisson distribution.

Now, we define,

$$
\Gamma_{n, k}=\left\{i=:\left\{i_{1}, i_{2}, \ldots, i_{k}\right\} \mid 1 \leq i_{1}<\ldots<i_{k} \leq n\right\}
$$

be the set of all possible combinations of $k$ vertices. we note that $C_{k}$ is a cycle of order $k$ in $\mathbb{G}(n, m, p)$ and say that $C_{k}$ is isolated in $\mathbb{G}(n, m, p)$ if there is no edge in $\mathbb{G}(n, m, p)$ between a vertex in the cycle and the other outside of the cycle.

For each $i \in \Gamma_{n, k}$, we define the indicator random variable

$$
X_{i}=\left\{\begin{aligned}
1 & \text { if there is an isolated cycle in } \mathbb{G}(n, m, p) \text { that spans the vertices } \\
& i=\left(i_{1}, \ldots, i_{k}\right), \\
0 & \text { otherwise, }
\end{aligned}\right.
$$


and set

$$
W_{n, k}=\sum_{i \in \Gamma_{n, k}} X_{i} .
$$

Thus, $W_{n, k}$ is the number of isolated cycles in $\mathbb{G}(n, m, p)$.

If $m=\left\lfloor n^{\alpha}\right\rfloor ; \alpha>0$, we demonstrate that $W_{n, k}$ can be approximated by Poisson approximation with parameter

$$
\begin{aligned}
\lambda:=\mathbb{E}\left(W_{n, k}\right)=\left(\begin{array}{l}
n \\
k
\end{array}\right) P\left(X_{i}=1\right) & =\left(\begin{array}{l}
n \\
k
\end{array}\right) \sum_{s=0}^{m}\left(\begin{array}{c}
m \\
s
\end{array}\right) p^{s+k}(1-p)^{m-s}(1-p)^{(n-k) s} \\
& =\left(\begin{array}{l}
n \\
k
\end{array}\right) p^{k}\left[1-p+p(1-p)^{(n-k)}\right]^{m} .
\end{aligned}
$$

By using Stein-Chen and Coupling Method. The main result is following,

Theorem 1.3. Let $W$ be the number of isolated cycles in a random intersection graph $\mathbb{G}(n, m, p)$. For $A \subseteq\{0,1,2, \ldots, n\}$ and $m=\left\lfloor n^{\alpha}\right\rfloor$ for some $\alpha>0$, then

$$
\left|P(W \in A)-P o i_{\lambda}(A)\right| \leq \frac{C_{(\lambda, A)}\left(1+\frac{n^{k}}{k !} k^{2}\right) p^{k}}{e^{m p\left(1-(1-p)^{n-2 k}\right)}}
$$

where $C_{(\lambda, A)}=\min \left\{1, \lambda, \frac{\triangle(\lambda)}{M_{A}+1}\right\}$,

$$
\triangle(\lambda)= \begin{cases}e^{\lambda}+\lambda-1 & \text { if } \lambda^{-1}\left(e^{\lambda}-1\right) \leq M_{A}, \\ 2\left(e^{\lambda}-1\right) & \text { if } \lambda^{-1}\left(e^{\lambda}-1\right)>M_{A},\end{cases}
$$

and

$$
M_{A}= \begin{cases}\max \left\{w \mid C_{w} \subseteq A\right\} & \text { if } 0 \in A, \\ \min \{w \mid w \in A\} & \text { if } 0 \notin A .\end{cases}
$$

When $C_{w}=\{0,1, \ldots, w\}$

Corollary 1.3. Let $W$ be the number of isolated cycles in a random intersection graph $\mathbb{G}(n, m, p)$. If $A \subseteq\{0,1,2, \ldots, n\}, m=\left\lfloor n^{\alpha}\right\rfloor$ for some $\alpha>0$, $q=1-p$ and $p=\frac{1}{n^{\gamma}}$ for any $\gamma \in \mathbb{R}^{+}\{1\}$, then

$$
\left|P(W \in A)-P o i_{\lambda}(A)\right| \leq \frac{C(\lambda, A)}{n^{2(\alpha-\gamma)\left(1-(q)^{n-2 k}\right)+(k \gamma-k)}},
$$

where $C(\lambda, A)=2 \min \left\{1, \lambda, \frac{\triangle(\lambda)}{M_{A}+1}\right\}$. 


\section{Stein-Chen and Coupling Method}

In 1972, Stein [1] gave a new technique to find a bound in the normal approximation to a distribution of a sum of dependent random variables. His technique was relied instead on the elementary differential equation. In 1975, Chen $[4,5]$ applied Stein's idea to the Poisson case. The central idea of the Stein-Chen method is the difference equation

$$
I_{A}(j)-\mathcal{P}_{\lambda}(A)=\lambda g_{\lambda, A}(j+1)-j g_{\lambda, A}(j), j \in \mathbb{N} \cup\{0\}
$$

where $\lambda>0$ and $A \subseteq \mathbb{N} \cup\{0\}$ and $I_{A}: \mathbb{N} \cup\{0\} \rightarrow \mathbb{R}$ is defined by

$$
I_{A}(w)= \begin{cases}1 & ; w \in A \\ 0 & ; w \notin A .\end{cases}
$$

The equation (3) is called Stein's equation for Poisson distribution function and its solution is

$$
g_{\lambda, A}(w)= \begin{cases}(w-1) ! \lambda^{-w} e^{\lambda}\left[\mathcal{P}_{\lambda}\left(I_{A \cap C_{w-1}}\right)-\mathcal{P}_{\lambda}\left(I_{A}\right) \mathcal{P}_{\lambda}\left(I_{C_{w-1}}\right)\right] & ; w \geq 1, \\ 0 & ; w=0\end{cases}
$$

where

$$
C_{w-1}=\{0,1, \ldots, w-1\} \text { and } \mathcal{P}_{\lambda}\left(I_{A}\right)=e^{-\lambda} \sum_{l=0}^{\infty} I_{A}(l) \frac{\lambda^{l}}{l !} .[7]
$$

By substituting $j$ and $\lambda$ in (3) by any integer-valued random variable $W$ and $\lambda=\mathrm{E}(W)$, we have

$$
P(W \in A)-P o i_{\lambda}(A)=\mathrm{E}\left(\lambda g_{\lambda, A}(W+1)\right)-\mathrm{E}\left(W g_{\lambda, A}(W)\right) .
$$

So far $W$ could be $\sum_{i \in \Gamma} X_{i}$ and $\lambda=\mathbb{E}(W)=\sum_{i \in \Gamma} p_{i}$ where $p_{i}=\mathbb{E}\left(X_{i}\right)=$ $P\left(X_{i}=1\right)$.

In 1992, Barbour, Holst and Janson[7] constructed coupling random variable $W_{i}$ and used Stein-Chen method to find the bound in Poisson approximation of $W$. They assumed that for each $i$ the distribution $L\left(W_{i}\right)$ of $W_{i}$ equals to the conditional distribution $L\left(W-X_{i} \mid X_{i}=1\right)$ and gave the fundamental theorem as follows:

Theorem 2.1. If $W$ and $W_{i}$ are defined as above, then

$$
\left|P(W \in A)-\operatorname{Poi}_{\lambda}(A)\right| \leq\left\|g_{\lambda, A}\right\| \sum_{i \in \Gamma} p_{i} \mathbb{E}\left(\left|W-W_{i}\right|\right)
$$

where $\left\|g_{\lambda, A}\right\|:=\sup _{w}\left[g_{\lambda, A}(w+1)-g_{\lambda, A}(w)\right]$. 
In 2006, Santiwipanont and Teerapabolarn [8] proved that for any subset $A$ of $\{0,1, \ldots, n\}$,

$$
\left\|g_{\lambda, A}\right\| \leq \lambda^{-1} \min \left\{1, \lambda, \frac{\triangle(\lambda)}{M_{A}+1}\right\}
$$

where

$$
\triangle(\lambda)= \begin{cases}e^{\lambda}+\lambda-1 & \text { if } \lambda^{-1}\left(e^{\lambda}-1\right) \leq M_{A}, \\ 2\left(e^{\lambda}-1\right) & \text { if } \lambda^{-1}\left(e^{\lambda}-1\right)>M_{A},\end{cases}
$$

and

$$
M_{A}= \begin{cases}\max \left\{w \mid C_{w} \subseteq A\right\} & \text { if } 0 \in A, \\ \min \{w \mid w \in A\} & \text { if } 0 \notin A .\end{cases}
$$

In next section, we will use Theorem 2.1 and (6) to prove our main result by constructing the random variable $W_{i}$.

\section{Proof of Main Result}

Let $A \subseteq \mathbb{N}$. By (5), it is enough to bound $\mathbb{E}\left|W-W_{i}\right|$ for $i \in \Gamma_{n, k}$ where the distribution of $W_{i}$ equals to the conditional distribution of $W-X_{i}$ given $X_{i}=1$.

Let $W_{i}$ be the number of isolated cycles of order $k$ in a random intersection graph $\mathbb{G}(n, m, p)-i, \mathbb{G}(n, m, p)-i$ obtained from $\mathbb{G}(n, m, p)$ by dropping the set $i \subseteq V$ and all the edges containing any of these vertices. Then for $w_{0} \in$ $\left\{0,1, \ldots,\left\lfloor\frac{n-k}{k}\right\rfloor\right\}$, we have

$$
\begin{aligned}
P\left(W_{i}=w_{0}\right) & =\left(\begin{array}{c}
n-k \\
k \\
w_{0}
\end{array}\right)\left[\sum_{s=0}^{m}\left(\begin{array}{c}
m \\
s
\end{array}\right) p^{s+k}(1-p)^{m-s}(1-p)^{(n-2 k) s}\right]^{w_{0}} \\
& =\left(\begin{array}{c}
n-k \\
k \\
w_{0}
\end{array}\right) p^{k w_{0}}\left[1-p+p(1-p)^{(n-2 k)}\right]^{m w_{0}}
\end{aligned}
$$

and

$$
\begin{aligned}
& P\left(W-X_{i}=w_{0} \mid X_{i}=1\right) \\
& =\frac{P\left(W-X_{i}=w_{0}, X_{i}=1\right)}{P\left(X_{i}=1\right)} \\
& =\frac{P\left(W=w_{0}+1, X_{i}=1\right)}{P\left(X_{i}=1\right)} \\
& =\frac{\left.\left(\begin{array}{c}
n-k \\
k \\
w_{0}
\end{array}\right)\right)\left[\sum_{s=0}^{m}\left(\begin{array}{c}
m \\
s
\end{array}\right) p^{s+k}(1-p)^{m-s}(1-p)^{(n-2 k) s}\right]^{w_{0}} \sum_{s=0}^{m}\left(\begin{array}{c}
m \\
s
\end{array}\right) p^{s+k}(1-p)^{m-s}(1-p)^{(n-2 k) s}}{\sum_{s=0}^{m}\left(\begin{array}{c}
m \\
s
\end{array}\right) p^{s+k}(1-p)^{m-s}(1-p)^{(n-2 k) s}} \\
& =\left(\begin{array}{c}
n-k \\
k \\
w_{0}
\end{array}\right)\left[\sum_{s=0}^{m}\left(\begin{array}{c}
m \\
s
\end{array}\right) p^{s+k}(1-p)^{m-s}(1-p)^{(n-2 k) s}\right]^{w_{0}}
\end{aligned}
$$




$$
=\left(\begin{array}{c}
\left(\begin{array}{c}
n-k \\
k
\end{array}\right) \\
w_{0}
\end{array}\right) p^{k w_{0}}\left[1-p+p(1-p)^{(n-2 k)}\right]^{m w_{0}} .
$$

From (7) and (8), the distribution of $W_{i}$ equals to the conditional distribution of $\left(W-X_{i} \mid X_{i}=1\right)$.

For $i, j \in \Gamma_{n, k}$ such that $i \neq j$, we define the indicator random variable $X_{j}^{(i)}$ and $E_{i j}$, as follow

$$
X_{j}^{(i)}=\left\{\begin{array}{l}
1 \quad \text { if here is an isolated cycle in } \mathbb{G}(n, m, p)-i \text { that } \\
\text { spans the vertices } i=\left(i_{1}, \ldots, i_{k}\right) \\
0 \quad \text { otherwise, }
\end{array}\right.
$$

and

$$
E_{i j}= \begin{cases}1 & \text { if there exists adjacent between } i_{k} \in i \text { and } j_{l} \in j \\ 0 & \text { otherwise. }\end{cases}
$$

We observe that in case $X_{i}=1$, that is we have an isolated cycle in $\mathbb{G}(n, m, p)$ that span vertices $i=\left\{i_{1}, \ldots, i_{k}\right\}$. Thus the number of isolated cycles in a random intersection graph $\mathbb{G}(n, m, p)-i$ equals to the number of isolated cycles in a random intersection graph $\mathbb{G}(n, m, p)$ minus 1 , that is

$$
W_{i}=W_{n, k}-1 \text {. }
$$

In case $X_{i}=0$. For $j \in \Gamma_{n, k}$ such that $j \cap i=\emptyset$ and $j \neq i$, then

$$
W_{i}=W_{n, k}+\sum_{j \in \Gamma_{n, k}, j \cap i=\emptyset} E_{i j} X_{j}^{(i)}
$$

that is the number of isolated cycles in $\mathbb{G}(n, m, p)-i$ equals to the sum of the number of isolated cycles in $\mathbb{G}(n, m, p)$ and the number of isolated cycles in $\mathbb{G}(n, m, p)-i$ which are connected to $i$.

We know that

$$
\left|W_{n, k}-W_{i}\right|=\left(W_{n, k}-W_{i}\right)^{+}+\left(W_{n, k}-W_{i}\right)^{-}
$$

where $\left(W_{n, k}-W_{i}\right)^{+}=\max \left\{W_{n, k}-W_{i}, 0\right\}$ and $\left(W_{n, k}-W_{i}\right)^{-}=-\min \left\{W_{n, k}-\right.$ $\left.W_{i}, 0\right\}$. Since $-\min \left\{W_{n, k}-W_{i}, 0\right\}=\max \left\{W_{i}-W_{n, k}, 0\right\}=\left(W_{i}-W_{n, k}\right)^{+}$, we have

$$
\mathbb{E}\left|W_{n, k}-W_{i}\right|=\mathrm{E}\left(W_{n, k}-W_{i}\right)^{+}+\mathrm{E}\left(W_{i}-W_{n, k}\right)^{+} .
$$

Form (9) and (10), we have 


$$
\begin{aligned}
\left(W_{n, k}-W_{i}\right)^{+} & \leq X_{i} \\
\left(W_{i}-W_{n, k}\right)^{+} & \leq \sum_{j \in \Gamma_{n, k}, j \cap i=\emptyset} E_{i j} X_{j}^{(i)} .
\end{aligned}
$$

We note that,

$$
\begin{aligned}
\mathbb{E}\left(X_{i}\right) & =\frac{\mathbb{E}\left(W_{n, k}\right)}{\left(\begin{array}{l}
n \\
k
\end{array}\right)} \\
& =\frac{\left(\begin{array}{c}
n \\
k
\end{array}\right) \sum_{s=0}^{m}\left(\begin{array}{c}
m \\
s
\end{array}\right) p^{s+k}(1-p)^{m-s}(1-p)^{(n-k) s}}{\left(\begin{array}{c}
n \\
k
\end{array}\right)} \\
& =\sum_{s=0}^{m}\left(\begin{array}{c}
m \\
s
\end{array}\right) p^{s+k}(1-p)^{m-s}(1-p)^{(n-k) s} \\
& =p^{k}\left[1-p+p(1-p)^{(n-k)}\right]^{m} .
\end{aligned}
$$

and

$$
\begin{aligned}
\sum_{j \in \Gamma_{n, k}, j \cap i=\emptyset} \mathrm{E}\left[E_{i j} X_{j}^{(i)}\right] & =\sum_{j \in D, j \cap i=\emptyset} P\left(E_{i j}=1, X_{j}^{(i)}=1\right) \\
& =\left(\begin{array}{c}
n-k \\
k
\end{array}\right)\left(1-q^{k^{2}}\right) \sum_{s=0}^{m}\left(\begin{array}{c}
m \\
s
\end{array}\right) p^{s+k}(1-p)^{m-s}(1-p)^{(n-2 k) s} \\
& \leq \frac{n^{k}}{k !} p^{k} k^{2}\left[1-p+p(1-p)^{(n-2 k)}\right]^{m}
\end{aligned}
$$

From (11), (12) and use the fact that $1-p \leq \frac{1}{e^{p}}$, we have

$$
\begin{aligned}
\mathbb{E}\left(\left|W-W_{i}\right|\right) & =\mathbb{E}\left(W-W_{i}\right)^{+}+\mathbb{E}\left(W_{i}-W\right)^{+} \\
& \leq \mathbb{E}\left(X_{i}\right)+\sum_{j \in \Gamma_{n, k}, j \cap i=\emptyset} \mathbb{E}\left(E_{i j} X_{j}^{(i)}\right) \\
& \leq p^{k}\left[1-p+p(1-p)^{(n-k)}\right]^{m}+\frac{n^{k}}{k !} p^{k} k^{2}\left[1-p+p(1-p)^{(n-2 k)}\right]^{m} \\
& \leq\left[1-p+p(1-p)^{(n-2 k)}\right]^{m}\left(1+\frac{n^{k}}{k !} k^{2}\right) p^{k} \\
& =\left[1-p\left(1-(1-p)^{(n-2 k)}\right]^{m}\left(1+\frac{n^{k}}{k !} k^{2}\right) p^{k}\right.
\end{aligned}
$$




$$
\leq \frac{\left(1+\frac{n^{k}}{k !} k^{2}\right) p^{k}}{e^{m p\left(1-(1-p)^{n-2 k}\right)}}
$$

Hence, by (5), (6) and (13), we have

$$
\left|P(W \in A)-P \operatorname{Poi}_{\lambda}(A)\right| \leq \frac{C_{(\lambda, A)}\left(1+\frac{n^{k}}{k !} k^{2}\right) p^{k}}{e^{m p\left(1-(1-p)^{n-2 k}\right)}}
$$

where $C_{(\lambda, A)}=\min \left\{1, \lambda, \frac{\triangle(\lambda)}{M_{A}+1}\right\}$.

This complete the proof of Theorem 1.3.

\section{Proof of Corollary $\mathbf{1 . 3 .}$}

From (13), we have

$$
\begin{aligned}
\mathbb{E}\left(\left|W-W_{i}\right|\right) & \leq \frac{\left(1+\frac{n^{k}}{k !} k^{2}\right) p^{k}}{e^{m p\left(1-(1-p)^{n-2 k}\right)}} \\
& =\frac{p^{k}}{e^{m p\left(1-(1-p)^{n-2 k}\right)}}+\frac{k(n p)^{k}}{(k-1) ! e^{m p\left(1-(1-p)^{n-2 k}\right)}}
\end{aligned}
$$

Suppose that $q=1-p$ and $m=\left\lfloor n^{\alpha}\right\rfloor$ for some $\alpha>0$ and $p=\frac{1}{n^{\gamma}}$ for any $\gamma \in \mathbb{R}^{+} \backslash\{1\}$, then we obtain

$$
\begin{aligned}
\mathbb{E}\left(\left|W-W_{i}\right|\right) & \leq \frac{1}{n^{(k) \gamma} e^{m p\left(1-(1-p)^{n-2 k}\right)}}+\frac{k}{(k-1) ! n^{(\gamma-1) k} e^{m p\left(1-(1-p)^{n-2 k}\right)}} \\
& \leq \frac{1}{n^{k \gamma} e^{m p\left(1-(1-p)^{n-2 k}\right)}}+\frac{n^{k} k}{(k-1) ! n^{k \gamma} e^{m p\left(1-(1-p)^{n-2 k}\right)}} \\
& \leq \frac{\left(1+n^{k}\right)}{n^{k \gamma} e^{m p\left(1-(1-p)^{n-2 k}\right)}} \\
& \leq \frac{2}{n^{(k \gamma-k)} e^{m p\left(1-(1-p)^{n-2 k}\right)}} \\
& \leq \frac{2}{n^{(k \gamma-k)} e^{n^{\alpha-\gamma}\left(1-(1-p)^{n-2 k}\right)}} \\
& \leq \frac{2}{n^{(k \gamma-k)} n^{2(\alpha-\gamma)\left(1-(q)^{n-2 k}\right)}} \\
& =\frac{2}{n^{2(\alpha-\gamma)\left(1-(q)^{n-2 k}\right)+(k \gamma-k)}}
\end{aligned}
$$


By (5), (6) and (15), we have

$$
\left|P(W \in A)-\operatorname{Poi}_{\lambda}(A)\right| \leq \frac{C(\lambda, A)}{n^{2(\alpha-\gamma)\left(1-(q)^{n-2 k}\right)+(k \gamma-k)}},
$$

where $C(\lambda, A)=2 \min \left\{1, \lambda, \frac{\triangle(\lambda)}{M_{A}+1}\right\}$.

This complete the proof of Corollary 1.3.

\section{Acknowledgments}

We are thankful for financial support, School of Science, University of Phayao, Thailand.

\section{References}

[1] A.D. Barbour and Louis H.Y. Chen, An introduction to stein's method, 2005, Singapore university Press and world scientific Publishing Co. Pte. Ltd.

[2] M. Karonski, E.R.Scheinerman and K.B.Singer-Cohen. On random intersection graphs: the subgraph problem, Combinatorics, Probability and Computing, Vol. 8: 131-159 (1999).

[3] Fill, J., Scheinerman, E. and Singer-Cohen, K. (2000): Random intersection graphs when $\mathrm{m}=\mathrm{w}(\mathrm{n})$ : an equivalence theorem relating the evolution of the G(n,m,p) and G(n,p) models, Random Structures Algorithms 16, 156-176. Lecture Note Ser., vol. 276, Cambridge University Press, Cambridge, 1999, pp. 239298.

[4] L.H.Y. Chen (1975), Poisson approximation for dependent trials, Annals of probability, 3:534-545.

[5] P. Erdős and A. Rényi. On the evolution of random graphs, Publ. Math. Inst. Hungar. Acad. Sci. 5 (1960) 1761.

[6] Y. Shang (2011), On the Isolated Vertices and Connectivity in Random Intersection Graphs, Hindawi Publishing Corporation International Journal of Combinatorics, Volume 2011, Article ID 872703.

[7] A.D. Barbour, L. Holst and S. Janson, Poisson approximation. Oxford Studies in probability 2, Clarendon Press, Oxford, 1992. 
[8] T. Santiwipanont and K. Teerapabolarn, Two formulas of non-uniform bounds on Poisson approximation for dependent indicators, Thai journal of mathematics (2006).

[9] M. Donganont, The bounds on Poisson Approximation of the number of isolated vertices in a random intersection graph, International Journal of Pure and Applied Mathematics, ISSN 1311-8080, Volume 87, No. 2 (2013).

[10] M. Donganont, Poisson Approximation of the number of isolated trees in a random intersection graph, International Journal of Pure and Applied Mathematics, ISSN 1311-8080, Volume 88, No. 1 (2013), 113-123. 